\title{
Uveal metastases in the mid-southeastern United States: a single-institution experience
}

This article was published in the following Dove Press journal:

Clinical Ophthalmology

\author{
Benjamin A King 1,2 \\ Elizabeth U Rosenberger' \\ Vanessa M Morales- \\ Tirado ${ }^{1,3}$ \\ Matthew W Wilson ${ }^{1,2}$ \\ 'Department of Ophthalmology, \\ Hamilton Eye Institute, University of \\ Tennessee Health Science Center, \\ College of Medicine, Memphis, \\ TN, USA; 'Department of Surgery, \\ St Jude Children's Research Hospital, \\ Memphis, TN, USA; ${ }^{3}$ Department \\ of Microbiology, Immunology, and \\ Biochemistry, University of Tennessee \\ Health Science Center, College of \\ Medicine, Memphis, TN, USA
}

Purpose: To report the clinical features of uveal metastases in a geographic region associated with high tobacco use.

Methods: Medical records from all patients diagnosed with uveal metastasis at a single tertiary referral center between 2000 and 2017 were retrospectively reviewed. The clinical features and the primary tumor site associated with each metastatic lesion were recorded.

Results: Ninety-nine uveal metastatic tumors were identified in 85 eyes of 74 patients ( 34 males). Median age at diagnosis was 62 years. Median tumor diameter was $11.6 \mathrm{~mm}$ and median height was $3.1 \mathrm{~mm}$. Carcinoma of the lung was the most common primary tumor occurring in 37 patients $(50 \%)$ followed by breast in 16 patients $(21 \%)$. Among females, metastatic lesions originated from the lung in 18 patients and from the breast in 16 patients. Median survival following intraocular metastasis was 9 months for patients with a primary lung malignancy and 36 months for patients with breast cancer (log-rank test, $P=0.002$ ).

Conclusion: Intraocular metastasis is more frequently observed in patients with carcinomas of the lung rather than breast at our treatment center. Both regional and global changes in cancer epidemiology most likely account for the findings in this study.

Keywords: iris, choroid, cancer, metastases, lung, breast, eye

\section{Introduction}

Carcinomas metastasize through lymphatics to regional nodal basins or hematogenously to more distant sites. Tissues with high vascular density such as the uvea are thus prone to metastases. Metastatic tumors involving the uveal tract represent the most common intraocular malignancy with an estimated annual incidence of $\sim 20,000^{1}$ in the USA. Prior large case series have consistently identified invasive breast carcinoma as the most common source of intraocular metastasis..$^{2-5}$

Since the first case series was published, epidemiologic trends have changed significantly and bronchogenic uveal metastases may now be more common than previously thought. Between 1975 and 2005, lung cancer incidence among US females increased $119 \%$ while the incidence of breast cancer remained relatively stagnant. ${ }^{6}$ Lung cancer mortality in women has now exceeded that from breast cancer in the $\mathrm{USA}^{7}$ and Europe ${ }^{8}$ since 1987 and 2015, respectively. Regional differences in cancer epidemiology may also increase the observed frequency of some metastatic lesions. In the USA, the prevalence of tobacco use is highest in the South and Midwest ${ }^{9}$ and these regions likewise suffer a higher incidence of lung cancer. Of those states with the highest percentage of smoking-related cancer mortality, the majority are located in the Southeast. ${ }^{10}$ Bronchogenic metastases may, therefore, be observed at disproportionately higher rates in these regions as well. Based on the available
Correspondence: Matthew W Wilson FACS, University of Tennessee Health Sciences Center, 930 Madison Avenue, Room 476, Memphis, TN 38163, USA

Tel +l 90I 4484283

Fax + I 90I 4485028

Email mwilson5@uthsc.edu 
information, however, the assumption that intraocular metastases most frequently arise from breast carcinoma remains unchallenged.

Understanding the relative differences between each primary malignancy associated with intraocular metastasis is especially important for clinicians as many of these lesions especially those associated with lung cancer ${ }^{2,3}$ - are recognized before the primary tumor, and the ophthalmologist is often responsible for initiating the diagnostic workup, which confirms disseminated metastatic disease. To investigate how regional variation in cancer epidemiology might affect the incidence of metastatic disease in the eye, we performed a retrospective case series examining patients with uveal metastases evaluated at a single tertiary care referral center covering the Mid-Southeastern USA.

\section{Methods}

Following approval by the institutional review board at the University of Tennessee Health Sciences Center, a billing code inquiry was requested on all patients treated at our center from 2000 until 2017 with the following International Classification of Diseases, Ninth Revision (ICD-9) or International Classification of Diseases, 10th Revision (ICD-10) codes corresponding with a malignant neoplasm of the choroid, ciliary body, or iris: 190.6, 190.0, C69.3, C69.4, C69.9. All patients subsequently treated for a primary ocular malignancy, such as uveal melanoma, were excluded. The remaining 658 patient records were then reviewed for the following inclusion criteria: 1) solid tumor involving the choroid, ciliary body, or iris with clinical features consistent with a metastatic lesion and 2) systemic workup confirming active metastatic cancer based on imaging or histopathology. All aspects of this retrospective review were compliant with the Health Insurance Portability and Accountability Act (HIPAA) and the Declaration of Helsinki, and therefore no consent was necessary to review patient data.

Prior medical history specific to the patient's cancer diagnosis was documented at the time of the initial examination and retrospectively reviewed to confirm the date of diagnosis, previous treatment, and status of disease. Baseline tumor characteristics including tumor size, laterality, and location were then documented based on clinical findings from the fundus examination and diagnostic imaging. Following the initial examination, medical records including imaging and biopsy reports from each patient's medical oncologist were reviewed to confirm the location and extent of disease. No patient required intraocular biopsy to confirm the diagnosis though histopathology was evaluated for enucleated specimens. Statistical analysis was performed with
SPSS 24 software (IBM Corporation, Armonk, NY, USA) or Microsoft Excel (Microsoft Corporation, Redmond, WA, USA) with $P$-values $<0.05$ defined as significant. Snellen visual acuity measurements were converted to logarithm of the minimum angle of resolution (log-MAR) for statistical analysis and the results were converted back to Snellen units. Patient mortality was retrospectively verified in the US Social Security Death Index. Median survival was calculated using Kaplan-Meier analysis.

\section{Results}

Uveal metastatic tumors were diagnosed in 74 patients 34 males and 40 females - during the study period. Patients were referred from six states in the Southeastern USA: 26 from Tennessee (35\%), 21 from Mississippi (28\%), 16 from Arkansas (22\%), 7 from Louisiana (9\%), 3 from Alabama (4\%), and 1 from Missouri (1.3\%). Median age was 62 years at the time intraocular metastasis was confirmed. The primary tumor of origin was known and established in the medical record prior to the diagnosis of the intraocular metastasis in 45 patients (61\%). Median time from diagnosis of the primary tumor to ocular metastasis was 23 months (range 0-188 months). In the remaining 29 patients (39\%), the ophthalmologist diagnosed the metastatic lesion before the primary cancer was diagnosed. Among patients with lung cancer, $24(65 \%)$ presented to the ophthalmologist before the primary tumor was recognized. By comparison, all 16(100\%) patients with breast cancer presented with a known cancer diagnosis. Sixty of 74 patients $(81 \%)$ presented with a chief complaint of decreased visual acuity. Of the remaining 14 patients, $6(8 \%)$ complained of floaters or photopsias without subjective vision loss, $6(8 \%)$ were asymptomatic and were referred following routine eye examination, and 2 (2.8\%) presented with isolated eye pain. Median Snellen visual acuity at the time of metastasis was diagnosed was 20/60. Thirty-six eyes $(42 \%)$ presented with visual acuity of $20 / 40$ or better. Thirty-two eyes (38\%) presented with 20/200 or worse.

Of the 74 patients with confirmed uveal metastasis, the primary tumor involved the lung in 37 patients $(50 \%)$ and the breast in 16 patients $(21 \%)$. Other primary cancers included carcinoma of unknown primary in three patients (4\%), renal cell carcinoma in three patients (4\%), colorectal carcinoma in three patients $(4 \%)$, carcinoid in two patients $(3 \%)$, sarcoma in two patients $(3 \%)$, parotid adenocarcinoma in two patients $(3 \%)$, cutaneous melanoma in two patients $(3 \%)$, esophageal carcinoma in one patient $(1.4 \%)$, thyroid carcinoma in one patient (1.4\%), endometrial carcinoma in one patient (1.4\%), and acute myeloid leukemia in one patient (1.4\%) (Figure 1). Lung cancer was the most common primary tumor of origin 


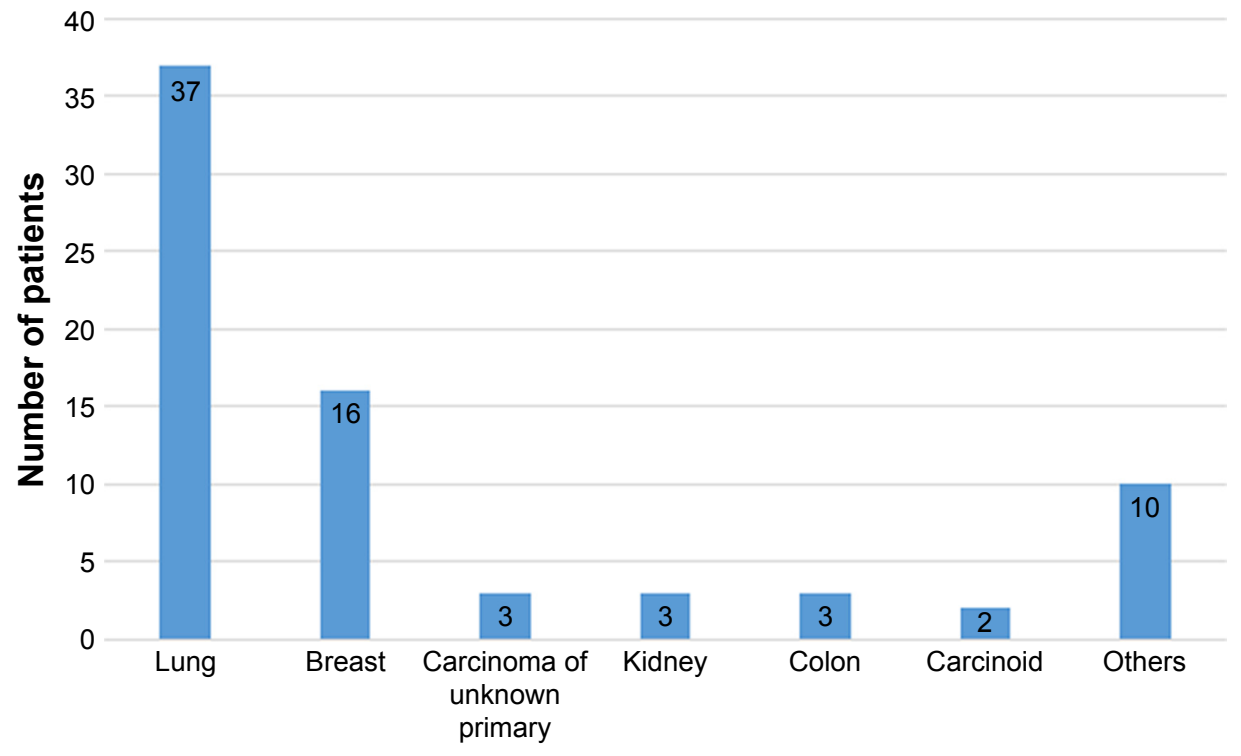

Figure I Uveal metastasis and primary tumor of origin.

among both male and female patients, accounting for 19 males (56\%) and 18 females (45\%) with uveal metastases (Table 1).

Seventeen of the 74 patients in this cohort presented with multiple metastatic lesions, including 11 patients with bilateral disease. Primary malignancies associated with bilateral metastases included breast cancer in six patients, lung cancer in two patients, endometrial carcinoma in one patient, renal cell carcinoma in one patient, and sarcoma in one patient. Bilateral metastases were more frequently observed among those with breast carcinoma ( $38 \%$ of patients) compared with only $9 \%$ of patients with other cancers (chi-squared test, $P=0.004$ ). A total of 99 intraocular metastases were observed in 85 eyes of 74 patients. The mean number of tumors occurring in each patient was 1.3 (range 1-8). Ninety-five lesions occurred in the choroid, three in the iris, and one in the

Table I Primary tumor of origin by gender

\begin{tabular}{lll}
\hline & $\begin{array}{l}\text { Males } \\
(\mathbf{n = 3 4 )}\end{array}$ & $\begin{array}{l}\text { Females } \\
(\mathbf{n}=\mathbf{4 0 )}\end{array}$ \\
\hline Lung & $\mathrm{I} 9(56 \%)$ & $\mathrm{I} 8(45 \%)$ \\
Breast & - & $\mathrm{I} 6(40 \%)$ \\
Carcinoma of unknown primary & $2(6 \%)$ & $\mathrm{I}(2.5 \%)$ \\
Kidney & $3(9 \%)$ & - \\
Colon & $2(6 \%)$ & $\mathrm{I}(2.5 \%)$ \\
Carcinoid & $\mathrm{I}(3 \%)$ & $\mathrm{I}(2.5 \%)$ \\
Sarcoma & $2(6 \%)$ & - \\
Parotid & $2(6 \%)$ & - \\
Esophagus & $\mathrm{I}(3 \%)$ & - \\
Thyroid & $\mathrm{I}(3 \%)$ & - \\
Uterus & - & $\mathrm{I}(2.5 \%)$ \\
Acute myeloid leukemia & - & $\mathrm{I}(2.5 \%)$ \\
Melanoma & $\mathrm{I}(3 \%)$ & $\mathrm{I}(2.5 \%)$ \\
\hline
\end{tabular}

ciliary body. Seventy-five of 95 choroidal tumors were large enough to delineate with B-scan ultrasonography. Median tumor diameter was $11.6 \mathrm{~mm}$ and median tumor thickness was $3.1 \mathrm{~mm}$.

Mortality was confirmed in 49 patients including 9 of 16 patients with breast cancer and 29 of 37 patients with lung cancer. Kaplan-Meier estimate of median survival following uveal metastasis was 15.0 months (95\% CI, 9.9-20.1 months). Median survival was significantly longer among patients with breast cancer at 36 months compared with 9 months among patients with lung cancer (log-rank test, $P=0.002$ ) (Figure 2).

\section{Discussion}

In this retrospective case series examining patients in the mid-southeastern USA, intraocular metastasis occurred

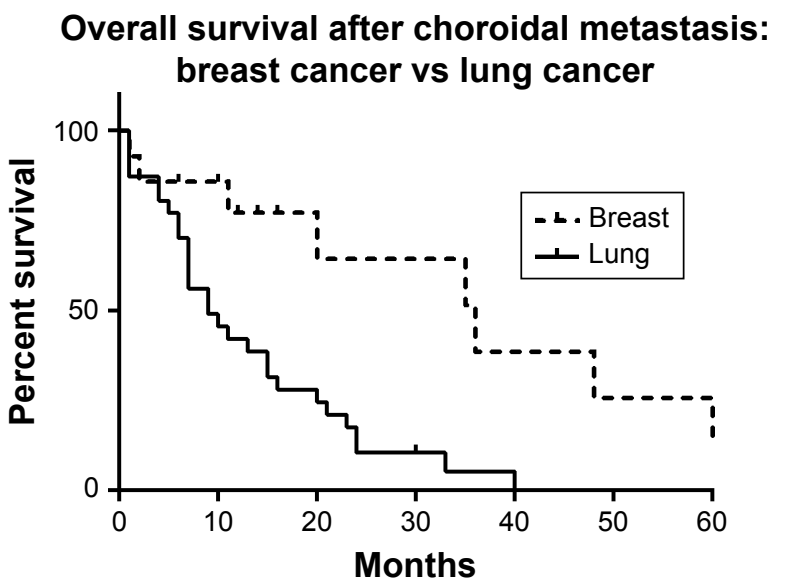

Figure 2 Kaplan-Meier survival analysis of mortality in patients with breast cancer and lung cancer following diagnosis of uveal metastasis. 
most frequently in the setting of lung cancer. This finding contradicts the current assumption that these lesions are more commonly associated with invasive breast carcinomas. Previous case series have reported that breast cancer accounts for between $40 \%$ and $50 \%$ of patients presenting with an intraocular metastasis while bronchogenic carcinomas only accounted for between $20 \%$ and $30 \%{ }^{2-5}$ These results were consistent among cohorts in the USA and the UK at four separate referral centers. Regional epidemiologic trends specific to our geographic area may account for the disparity between our results and those previously reported.

In 2014, invasive breast carcinoma was the most frequently diagnosed form of cancer in the USA. ${ }^{11}$ Within the six states represented in this case series, however, the average age-adjusted incidence of lung cancer exceeded that of breast cancer. Disproportionately higher rates of lung cancer within our geographic region ${ }^{11}$ may account for why bronchogenic metastases were more frequently observed in this cohort. It should be noted, however, that another regional case series in the Midwestern USA - an area with even higher prevalence of tobacco use ${ }^{9}$ - was conducted between 1964 and 1986 and still observed a substantially greater proportion of breast metastases. ${ }^{5}$

It should, therefore, be considered that our results may be more attributable to the recent global spike in lung cancer rates among females. In the USA, age-adjusted incidence of lung cancer in women more than doubled over the last four decades. ${ }^{6}$ In the European Union, female lung cancer rates continue to increase and have even exceeded that of males in certain countries. ${ }^{12}$ The three largest case series of uveal metastases in the USA collectively studied patients treated between 1958 and 1994,2,4,5 long before these global epidemiologic trends became apparent. Further investigation is required to confirm if the increased frequency of bronchogenic metastases which we observed is reproducible in other patient populations.

\section{Limitations}

The greatest limitation of this study is the presence of selection bias. Patients described here do not necessarily represent every diagnosed case of intraocular metastasis occurring within our region but rather those who were referred from an extensive network of retina specialists. Lung metastases may have a higher probability of referral as most present without a known cancer history and present a greater diagnostic challenge to referring providers. This referral bias may exaggerate the observed disparity in incidence between breast and lung metastases. The same referral bias was also present in other previously cited case series and therefore cannot fully account for the difference in our observations. It should also be considered that our findings only represent a single region within the USA and it is not clear to what extent the increased observation of bronchogenic metastases is due to regional variation, in cancer epidemiology or global trends, secondary to rising lung cancer incidence among women.

The clinical findings described in this investigation are otherwise consistent with those reported in previous case series. Metastatic lesions from breast cancer were more likely to be bilateral and multifocal as reported by Shields et $\mathrm{al}^{2}$ and Konstantinidis et al. ${ }^{3}$ Median survival after intraocular metastasis was significantly shorter among patients with lung cancer compared with those with breast cancer as was previously described by Freedman et al. ${ }^{5}$ Median survival for the entire cohort at 20 months was more than double that reported in a 1974 case series, ${ }^{4}$ reflecting a generalized decrease in overall cancer mortality. We also demonstrated that the majority of bronchogenic metastases come to presentation before the primary lung tumor is diagnosed, a trend also noted by Shields et $\mathrm{al}^{2}$ and Konstantinidis et al. ${ }^{3}$ This is arguably the most clinically relevant observation as it emphasizes the critical responsibility of the ophthalmologist in initiating the proper systemic workup for patients with occult lung malignancy.

\section{Conclusion}

In conclusion, metastatic intraocular tumors likely occur more frequently in association with bronchogenic carcinomas than previously thought. Local variation in the prevalence of tobacco use and lung cancer as well as the recent increase in lung cancer rates among females likely accounts for this. Further investigation is needed to confirm if these observations are reproducible in other geographic regions and patient populations.

\section{Acknowledgments}

This paper was presented at the American Association of Ophthalmic Oncologists and Pathologists (AAOOP) 2017 Annual Meeting, New Orleans, LA, November 10, 2017. Unrestricted Grant, Research to Prevent Blindness (New York, NY, USA).

\section{Disclosure}

The authors report no conflicts of interest in this work.

\section{References}

1. Cohen VM. Ocular metastases. Eye. 2013;27(2):137-141. 
2. Shields CL, Shields JA, Gross NE, Schwartz GP, Lally SE. Survey of 520 eyes with uveal metastases. Ophthalmology. 1997;104(8): 1265-1276.

3. Konstantinidis L, Rospond-Kubiak I, Zeolite I, et al. Management of patients with uveal metastases at the Liverpool Ocular Oncology Centre. Br J Ophthalmol. 2014;98(1):92-98.

4. Ferry AP, Font RL. Carcinoma metastatic to the eye and orbit: a clinicopathologic study of 227 cases. Arch Ophthalmol. 1974;92(4): 276-286.

5. Freedman MI, Folk JC. Metastatic tumors to the eye and orbit. Patient survival and clinical characteristics. Arch Ophthalmol. 1987;105(9): 1215-1219.

6. Howlader N, Noone AM, Krapcho M, et al. SEER Cancer Statistics Review, 1975-2014, National Cancer Institute. Bethesda, MD, Available from: https://seer.cancer.gov/csr/1975_2014/, based on November 2016 SEER data submission, posted to the SEER web site, April 29, 2017.

7. Egleston BL, Meireles SI, Flieder DB, Clapper ML. Populationbased trends in lung cancer incidence in women. Semin Oncol. 2009; 36(6):506-515.

8. Bosetti C, Malvezzi M, Rosso T, et al. Lung cancer mortality in European women: trends and predictions. Lung Cancer. 2012;78(3):171-178.
9. Current Tobacco Use Among U.S Adults Aged 18 Years and Older by Geographic Region. Reprinted from Centers for Disease Control and Prevention (CDC). Tobacco Use by Geographic Region. Atlanta, GA: U.S. Department of Health and Human Services, Centers for Disease Control and Prevention, National Center for Chronic Disease Prevention and Health Promotion, Office on Smoking and Health; 2017.

10. Lortet-Tieulent J, Goding Sauer A, Siegel RL, et al. State-level cancer mortality attributable to cigarette smoking in the United States. JAMA Intern Med. 2016;176(12):1792-1798.

11. Age-Adjusted Invasive Cancer Incidence Rates in the United States, 2014-2014. Data released June 12, 2017. Based on the December 2016 Call For Data Submission. Cancer-Rates.info. CINA+ Online Cancer in North America. Available from http://cancer-rates.info/naaccr/. Accessed November 21, 2017.

12. Lortet-Tieulent J, Renteria E, Sharp L, et al. Convergence of decreasing male and increasing female incidence rates in major tobaccorelated cancers in Europe in 1988-2010. Eur J Cancer. 2015;51(9): 1144-1163.
Clinical Ophthalmology

\section{Publish your work in this journal}

Clinical Ophthalmology is an international, peer-reviewed journal covering all subspecialties within ophthalmology. Key topics include: Optometry; Visual science; Pharmacology and drug therapy in eye diseases; Basic Sciences; Primary and Secondary eye care; Patien Safety and Quality of Care Improvements. This journal is indexed on

Submit your manuscript here: http://www.dovepress.com/clinical-ophthalmology-journal

\section{Dovepress}

PubMed Central and CAS, and is the official journal of The Society of Clinical Ophthalmology (SCO). The manuscript management system is completely online and includes a very quick and fair peer-review system, which is all easy to use. Visit http://www.dovepress.com/ testimonials.php to read real quotes from published authors. 